\title{
Hemodynamic Status During Endovascular Stroke Treatment: Association of Blood Pressure with Functional Outcome
}

\author{
Min Chen ${ }^{1 *} \mathbb{0}$, Dorothea Kronsteiner ${ }^{2}$, Johannes Pfaff ${ }^{3}$, Simon Schieber ${ }^{1}$, Laura Jäger ${ }^{1}$, Martin Bendszus ${ }^{3}$, \\ Meinhard Kieser ${ }^{2}$, Markus A. Möhlenbruch ${ }^{3}$, Peter A. Ringleb' ${ }^{1}$, Julian Bösel ${ }^{4}$ and Silvia Schönenberger ${ }^{1}$
}

(c) 2021 The Author(s)

\begin{abstract}
Background: Optimal blood pressure (BP) management during endovascular stroke treatment in patients with large-vessel occlusion is not well established. We aimed to investigate associations of BP during different phases of endovascular therapy with reperfusion and functional outcome.

Methods: We performed a post hoc analysis of a single-center prospective study that evaluated a new simplified procedural sedation standard during endovascular therapy (Keep Evaluating Protocol Simplification in Managing Periinterventional Light Sedation for Endovascular Stroke Treatment). BP during endovascular therapy in patients was managed according to protocol. Data from four different phases (baseline, pre-recanalization, post recanalization, and post intervention) were obtained, and mean BP values, as well as changes in BP between different phases and reductions in systolic BP (SBP) and mean arterial pressure (MAP) from baseline to pre-recanalization, were used as exposure variables. The main outcome was a modified Rankin Scale score of $0-2$ three months after admission. Secondary outcomes were successful reperfusion and change in the National Institutes of Health Stroke Scale score after $24 \mathrm{~h}$. Multivariable linear and logistic regression models were used for statistical analysis.

Results: Functional outcomes were analyzed in 139 patients with successful reperfusion (defined as thrombolysis in cerebral infarction grade $2 \mathrm{~b}-3$ ). The mean (standard deviation) age was 76 (10.9) years, the mean (standard deviation) National Institutes of Health Stroke Scale score was 14.3 (7.5), and 70 (43.5\%) patients had a left-sided vessel occlusion. Favorable functional outcome (modified Rankin Scale score 0-2) was less likely with every $10-\mathrm{mm} \mathrm{Hg}$ increase in baseline (odds ratio [OR] 0.76, $P=0.04$ ) and pre-recanalization (OR 0.65, $P=0.011$ ) SBP. This was also found for baseline $(\mathrm{OR} 0.76, P=0.05)$ and pre-recanalization MAP $(\mathrm{OR} 0.66, P=0.03)$. The maximum Youden index in a receiver operating characteristics analysis revealed an SBP of $163 \mathrm{~mm} \mathrm{Hg}$ and MAP of $117 \mathrm{~mm} \mathrm{Hg}$ as discriminatory thresholds during the pre-recanalization phase to predict functional outcome.

Conclusions: In our protocol-based setting, intraprocedural pre-recanalization BP reductions during endovascular therapy were not associated with functional outcome. However, higher intraprocedural pre-recanalization SBP and MAP were associated with worse functional outcome. Prospective randomized controlled studies are needed to determine whether BP is a feasible treatment target for the modification of outcomes.
\end{abstract}

Keywords: Acute ischemic stroke, Thrombectomy, Blood pressure

\footnotetext{
*Correspondence: min.chen@med.uni-heidelberg.de

1 Department of Neurology, Heidelberg University Hospital, Heidelberg, Germany

Full list of author information is available at the end of the article
}

\section{Introduction}

Today, the periinterventional, or at least the postinterventional, care of endovascular stroke treatment (EST) is increasingly managed by specialized physicians with 
neuroanesthetic expertise. Optimal blood pressure (BP) management during acute EST is not well established. Current guidelines recommend maintaining the systolic BP (SBP) under 180-185 mm Hg and above $140 \mathrm{~mm} \mathrm{Hg}$, as well as avoiding excessive BP drops during thrombectomy, with low to moderate levels of evidence [1-4].

Extreme levels of BP during an acute ischemic stroke may be detrimental, with a U-shaped relation between $\mathrm{BP}$ and functional outcome $[5,6]$. During the acute phase of ischemic stroke, BP is frequently elevated, which might constitute a necessary compensatory mechanism for maintaining cerebral perfusion $[7,8]$. On the other hand, maladaptively high systemic BP might lead to cerebral edema and hemorrhage [5].

EST has now become the recommended treatment for acute ischemic stroke due to large-vessel occlusion [9]. During EST, sedating medications are often used during the intervention, e.g., because of agitation, and their hemodynamic effect has to be monitored and, possibly, treated.

In this post hoc analysis of the Keep Evaluating Protocol Simplification in Managing Periinterventional Light Sedation for Endovascular Stroke Treatment (KEEP SIMPLEST) study [10], we aimed to identify potential associations of BP variations during four different phases of EST with short-term and long-term functional outcome and reperfusion in the setting of procedural sedation and with protocol-based BP management.

\section{Methods}

\section{Design of the KEEP SIMPLEST Study}

Keep Evaluating Protocol Simplification in Managing Periinterventional Light Sedation for Endovascular Stroke Treatment [10] was a prospective observational single-center study comparing the outcome of patients undergoing EST in procedural sedation under an optimized, lean standard operating procedure (SOP), with the preceding SOP in the procedural sedation arm of the Sedation vs. Intubation for Endovascular Stroke Treatment (SIESTA) trial [11]. To compare both SOPs, a propensity score matching approach was applied to the study cohort and the historical SIESTA cohort. Briefly, the new standard comprised managing patients preferentially in procedural sedation. When sedation was deemed necessary (by the interventionalist and/or neuroanesthetist), propofol and esketamine were initiated as short-acting sedatives and administered fractionized and/or continuously. Esketamine was used as a sedative to reduce the need for catecholamines and opioids. If patients still showed signs of pain, remifentanil for analgesia was applied per infusion. Applied doses are shown in Supplementary Table 1. A small proportion of patients (8.8\%) did not need sedative medication intraprocedurally.
Endotracheal intubation was performed when patients remained agitated or showed symptoms of respiratory insufficiency or when airway patency was lost. Furthermore, different simplified operational optimizations, such as better and faster communication sequences and the improvement of equipment by introducing a new mobile EST cart, were implemented in the new SOP. In our center, periinterventional care is performed by neurologists trained in monitored neuroanesthetic care.

The BP protocol specified an SBP target between 140 and $160 \mathrm{~mm} \mathrm{Hg}$ and an avoidance of values less than 120 or more than $180 \mathrm{~mm} \mathrm{Hg}$ during the pre-recanalization and post-recanalization phases. This target range has been chosen because retrospective studies suggested that an SBP less than $140 \mathrm{~mm} \mathrm{Hg}$ is associated with a worse functional outcome [12,13]; on the other hand, very high SBP [14] levels are also associated with a poor outcome.

The sample size was calculated after an interim analysis and led to the inclusion of 161 patients, of whom 69 were paired with the historic cohort of the SIESTA trial via propensity score matching.

Main clinical and procedural findings of KEEP SIMPLEST showed that early neurological improvement and modified Rankin Scale (mRS) scores at 3 months were not different compared with the preceding approach in the procedural sedation group of SIESTA, but operational advantages, such as reduced in-house treatment times, were achieved [10].

The study was approved by the local institutional review board (Ethikkommission Medizinische Fakultät Heidelberg, ID S-325/2015). There was no external funding for this study.

\section{Data Collection}

Data from 161 patients were collected in the KEEP SIMPLEST study from December 2016 to November 2017. To investigate possible associations between $\mathrm{BP}$ values and reductions from baseline and different outcome parameters, we decided to only analyze the subset of 139 patients with successful recanalization (defined as thrombolysis in cerebral infarction [TICI] grade $2 \mathrm{~b}-3$ ) for early neurological improvement (i.e., change in National Institutes of Health Stroke Scale [NIHSS] score after $24 \mathrm{~h}$ ) and an mRS score at 3 months because reperfusion status has a large influence on clinical outcomes and we chose to avoid its confounding effects [15]. To evaluate whether $\mathrm{BP}$ during the baseline and pre-recanalization phases has an influence on the reperfusion status, mean SBP, mean diastolic BP (DBP), and average mean arterial pressure (MAP) data were analyzed in the total study population. $\mathrm{BP}$ was measured noninvasively.

The Alberta stroke program early computed tomography score (ASPECTS) was routinely assessed 
preinterventionally in non-contrast-enhanced computed tomograpy imaging. ASPECTS and reperfusion status were collected via a chart review. The mRS score at 3 months was obtained via a telephone interview, and NIHSS scores at admission and $24 \mathrm{~h}$ after admission were obtained via a chart review.

Different hemodynamic and physiologic parameters, such as SBP, DBP, heart rate, peripheral oxygen saturation, and use of analgesics, sedatives, or catecholamines and their respective doses, were recorded. MAP values were calculated as $\mathrm{MAP}=(\mathrm{SBP}+2 \times \mathrm{DBP}) / 3$. All three $\mathrm{BP}$ dimensions (SBP, DBP, and MAP) were used for analysis. We defined four different periinterventional time segments: baseline, pre-recanalization, post recanalization, and post intervention. The segment after groin puncture and before the final recanalization result was specified as the pre-recanalization phase, and afterward as the postrecanalization phase. The mean of up to three BP measurements obtained in the emergency department was specified as the baseline value (baseline $\mathrm{BP}$ ), and if that value was not recorded, then $\mathrm{BP}$ values during the ambulance transport to the emergency department or shortly before the groin puncture were used. After groin puncture, BP was measured noninvasively every 5 min during the EST. We obtained the postinterventional BP values (postintervention BP) from the first three documented noninvasive or invasive BP measurements at the stroke unit or the intensive care unit.

Additionally, we chose to investigate whether a reduction in SBP or MAP of at least $20 \%, 30 \%$, or $40 \%$ from baseline to pre-recanalization was associated with unfavorable functional outcomes at 3 months. A reduction was defined when at least one of the 5-min interval measurements showed a value that was at least $20 \%, 30 \%$, or $40 \%$ lower than the mean baseline BP value.

Doses of bolus injections and perfusion rates for propofol and esketamine, as well as perfusion rates of remifentanil and norepinephrine, were documented for every 5 -min interval.

\section{Exposures}

The exposure variables were mean SBP, mean DBP, and mean MAP; changes in mean SBP, mean DBP, and mean MAP between the different phases of EST (from baseline to pre-recanalization, baseline to post recanalization, and baseline to post intervention); and reductions (of more than $20 \%, 30 \%$, and $40 \%$ ) in SBP and MAP compared with baseline values.

\section{Outcomes}

The primary outcome was the mRS score after 3 months. Secondary outcomes were change in NIHSS score after $24 \mathrm{~h}$ and reperfusion status.

\section{Statistical analysis}

Descriptive statistics were calculated for baseline and follow-up data, including mean and standard deviation (SD) for continuous data, median and interquartile range for scores, and absolute and relative frequencies for categorical data. The BP values were evaluated by the within-individual mean and SD.

The analysis aimed to evaluate the influence of changes between baseline BP and BP in the following phases, as well as mean BP during each phase, on the outcomes. We used linear and logistic regression models for recanalization status (TICI grade $0-2 \mathrm{a}$ vs. $2 \mathrm{~b}-3)$, change in NIHSS score at $24 \mathrm{~h}$, and mRS score at 3 months (0-2 vs. 3-6). Either mean SBP, mean DBP and mean MAP or their respective changes between different phases were included as predictors. Furthermore, the heart rate, baseline NIHSS score, preinterventional ASPECTS, intubation, and medication (propofol, norepinephrine, and esketamine) were included as predictor variables. In models for BP differences between different phases and mRS score, we omitted the medication because the adjustment led to unstable models. In addition, the association between BP drops of $20 \%$, $30 \%$, and $40 \%$ and the functional outcomes (change in NIHSS and mRS scores) was evaluated using linear and logistic regression models. Adjusted regression coefficients for linear regression models, or odds ratios (OR) for logistic regression models, with the corresponding confidence intervals (CI) and $P$ values are reported. Multiple imputation using chained equations generating 50 data sets and Rubin's rules are used to handle missing values [16]. To identify thresholds in SBP and MAP to predict good functional outcome (mRS score at 3 months $0-2$ vs. 3-6), a receiver operating characteristics (ROC) analysis was used. The thresholds were identified by maximizing the Youden index. The ROC curves, including the area under the ROC curve, and the corresponding sensitivity, specificity, and thresholds are reported.

Because this is an exploratory study, all $P$ values are of a descriptive nature, and no adjustment for multiple testing was applied.

The software R (version 4.0.2) was used for statistical analysis [17].

\section{Data Collection Statement}

The data that support the findings of this study are available from the corresponding author on reasonable request. 


\section{Results}

\section{Patient Characteristics}

Data from 161 patients were obtained in total. Successful recanalization (TICI grade $2 \mathrm{~b}-3$ ) was achieved in 139 patients $(86.4 \%)$.

In patients who were successfully recanalized, the mean (SD) age was 76 (10.9) years, 59\% were female, and the mean (SD) baseline NIHSS score was 14.3 (7.5). Eightyone $(58.3 \%)$ patients received alteplase prior to EST, and $52(37.4 \%)$ patients received EST only. Further baseline and outcome characteristics of patients with successful reperfusion are displayed in Table 1 . Baseline, procedural, and outcome characteristics of the total study population can be found in Supplementary Table 1.

Mean SBP, mean DBP, and mean MAP (SD) at baseline were 169.5 (24.5), 92.7 (15.7), and 118.3 (16.5) mm $\mathrm{Hg}$, respectively, which were subsequently lower in each phase of the intervention and post intervention (Table 2 and Supplementary Figure 1). Pre-recanalization MAP reductions of at least $20 \%$ occurred in $49.3 \%$ of patients (Table 1). The mean BP values of patients who were not sufficiently recanalized are shown in Supplementary Table 3.

\section{Association of BP with Reperfusion Status}

Baseline and pre-recanalization SBP, DBP, and MAP were not associated with the rate of successful reperfusion (Supplementary Table 4).

\section{Association of BP with Early Neurological Improvement}

The mean (SD) change in the NIHSS score after $24 \mathrm{~h}$ was -3.8 (9.0). A higher SBP during baseline $(\beta=0.12$ [95\% CI 0.04-0.19], $P=0.002)$, pre-recanalization $(\beta=0.18$ [95\% CI 0.08-0.29], $P<0.001)$, and post recanalization $(\beta=0.12$ [95\% CI $0.03-0.20], P=0.006)$, as well as a lower pre-recanalization DBP $(\beta=-0.19[95 \%$ $\mathrm{CI}-0.36$ to -0.02 ], $P=0.03)$, was significantly associated with less improvement of the NIHSS score at $24 \mathrm{~h}$ after admission. Every 10-mm $\mathrm{Hg}$ increase in baseline, pre-recanalization, and post-recanalization SBP led to 1.2, 1.8, and 1.2 less improvement in the NIHSS score, respectively.

Higher MAP during baseline $(\beta=0.09$ [95\% CI $0.00-$ 0.18 ], $P=0.063$ ) showed a trend for association with less improvement in the NIHSS score at $24 \mathrm{~h}$ after admission. Mean BP values in other phases showed no association with change in the NIHSS score after $24 \mathrm{~h}$ (Table 3 and Supplementary Table 5).

In the analysis of SBP, DBP, and MAP change between the baseline phase and the pre-recanalization, postrecanalization, and postintervention phases, only the degree of decrease in MAP from the baseline to the
Table 1 Demographic, baseline, procedural, and outcome characteristics

\begin{tabular}{|c|c|}
\hline & $n(\%)$ \\
\hline \multicolumn{2}{|l|}{ Demographic characteristics $(n=139)$} \\
\hline Age, mean (SD) (years) & $76.0(10.9)$ \\
\hline Female sex & $82(59.0)$ \\
\hline \multicolumn{2}{|l|}{ Premedication } \\
\hline Antiplatelet therapy & $50(36.0)$ \\
\hline Oral anticoagulant therapy & $22(15.8)$ \\
\hline Statin & $39(28.1)$ \\
\hline \multicolumn{2}{|l|}{ Vascular risk factors } \\
\hline Hypertension & $115(82.7)$ \\
\hline Diabetes mellitus & $34(24.5)$ \\
\hline Hyperlipidemia & $37(26.6)$ \\
\hline Smoking & $22(15.8)$ \\
\hline Atrial fibrillation & $46(33.1)$ \\
\hline Peripheral artery occlusive disease & $14(10.1)$ \\
\hline \multicolumn{2}{|l|}{ Premorbid mRS score } \\
\hline 0 & $47(33.8)$ \\
\hline 1 & $34(24.5)$ \\
\hline 2 & $20(14.4)$ \\
\hline$>2$ & $38(27.3)$ \\
\hline \multicolumn{2}{|l|}{ NIHSS score } \\
\hline Mean (SD) & $14.3(7.5)$ \\
\hline Median (IQR) & $14.0(8-20)$ \\
\hline \multicolumn{2}{|l|}{ Site of arterial occlusion } \\
\hline ICA occlusion & $43(27.2)$ \\
\hline MCA occlusion & $95(68.3)$ \\
\hline ICA+ MCA occlusion & 38 (23.6) \\
\hline Left side & $70(43.5)$ \\
\hline \multicolumn{2}{|l|}{ Treatment } \\
\hline Alteplase only & $6(4.3)$ \\
\hline Alteplase + EST & $81(58.3)$ \\
\hline EST only & $52(37.4)$ \\
\hline Door-to-needle time, mean (SD) ${ }^{a}(\mathrm{~min})$ & $32.0(13.0)$ \\
\hline \multicolumn{2}{|l|}{ Pretreatment ASPECTS ${ }^{b}$} \\
\hline $8-10$ & $89(65.9)$ \\
\hline $6-7$ & $31(23.0)$ \\
\hline$<6$ & $15(11.1)$ \\
\hline Median (IQR) & $8(7-10)$ \\
\hline \multicolumn{2}{|l|}{ Periinterventional aspects } \\
\hline Conversion to general anesthesia $^{c}$ & $14(10.1)$ \\
\hline Need of sedation & $127(91.4)$ \\
\hline Need of catecholamines & $43(30.9)$ \\
\hline MAP reduction of at least $20 \%^{d}$ & $67(49.3)$ \\
\hline MAP reduction of at least $30 \%^{\mathrm{d}}$ & $33(24.3)$ \\
\hline MAP reduction of at least $40 \%^{d}$ & $13(9.6)$ \\
\hline $\mathrm{MAP}<70 \mathrm{~mm} \mathrm{Hg}$ & $7(5.0)$ \\
\hline \multicolumn{2}{|l|}{ Functional outcome parameters $(n=139)$} \\
\hline NIHSS score change after $24 \mathrm{~h}$, mean (SD) & $-3.8(9.0)$ \\
\hline \multicolumn{2}{|l|}{ mRS score after 3 mo } \\
\hline $0-1$ & $37(26.6)$ \\
\hline
\end{tabular}


Table 1 (continued)

\begin{tabular}{|c|c|}
\hline & $n(\%)$ \\
\hline $0-2$ & $77(55.4)$ \\
\hline $0-3$ & $80(57.6)$ \\
\hline In-house mortality & $10(7.2)$ \\
\hline Mortality after 3 mo & $28(20.1)$ \\
\hline \multicolumn{2}{|c|}{ Degree of reperfusion $\left(\mathrm{TICl}\right.$ grade) $\left(n=161^{\mathrm{e}}\right)$} \\
\hline $0-2 a$ & $22(13.6)$ \\
\hline $2 b$ & $70(43.5)$ \\
\hline $2 c$ & $19(11.8)$ \\
\hline 3 & $50(31.1)$ \\
\hline
\end{tabular}

ASPECTS, Alberta stroke program early computed tomographic score, EST, endovascular stroke treatment, ICA, internal carotid artery, IQR, interquartile range, MAP, mean arterial pressure, MCA, middle cerebral artery, mRS, modified Rankin Scale, NIHSS, National Institutes of Health Stroke Scale, SD, standard deviation, $\mathrm{TICl}$, thrombolysis in cerebral infarction

a Data are missing in 41 patients

b ASPECTS were missing in 4 patients

c Reasons for conversion are shown in Supplementary Table 2

d Data are missing in 3 patients

e Data from the whole study population are shown here

postintervention phase was associated with less improvement in the NIHSS score $(\beta=0.09$ [95\% CI 0.01-0.17], $P=0.036)$. With every $10-\mathrm{mm} \mathrm{Hg}$ decrease, improvement in the NIHSS score was decreased by 0.9 (Table 3 and Supplementary Table 6).

Reductions of more than $20 \%, 30 \%$, or $40 \%$ in SBP and MAP from baseline during pre-recanalization showed no association with change in the NIHSS score after $24 \mathrm{~h}$ in a multivariable regression analysis (Supplementary Table 7).

\section{Association of BP with mRS Score $\mathbf{0 - 2}$ at $\mathbf{3}$ Months}

Seventy-seven (55.4\%) patients who received successful reperfusion had a favorable long-term functional outcome, defined as an mRS score of $0-2$ after 3 months (Table 1). For every $10-\mathrm{mm} \mathrm{Hg}$ increase in baseline and pre-recanalization SBP, there was a lower chance of achieving an mRS score of $0-2$ at 3 months, with ORs of $0.76(95 \%$ CI $0.61-0.95, P=0.015)$ and $0.65(95 \%$ CI $0.47-0.91, P=0.011)$, respectively. This was also seen for baseline MAP (OR 0.76 [95\% CI 0.58-1.00], $P=0.05$ ) and pre-recanalization MAP (OR 0.66 [95\% CI 0.45-0.96], $P=0.03$ ).

Absolute mean BP values in other phases showed no association with the mRS score at 3 months (Table 4 and Supplementary Table 8).

When the changes in mean SBP, mean MAP, and mean DBP from baseline to pre-recanalization, post recanalization, and post intervention were analyzed, only the decrease in MAP from baseline to post intervention was associated with an mRS score of 0-2 at 3 months. The odds of achieving an mRS score of 0-2 was lower in the group with a larger MAP decrease, with an OR of 0.75 (95\% CI $0.58-0.98, P=0.032)$ for every $10-\mathrm{mm} \mathrm{Hg}$ decrease (Table 4 and Supplementary Table 9).

A reduction of more than $20 \%, 30 \%$, or $40 \%$ in SBP or MAP from baseline during pre-recanalization was not associated with the mRS score at 3 months (Supplementary Table 7).

Threshold values for baseline SBP and MAP discriminating between mRS scores of 0-2 and 3-6 (see Fig. 1) were found to be 187 and $128 \mathrm{~mm} \mathrm{Hg}$, respectively, with lower values predicting an mRS score of $0-2$. The areas under the curves were 0.64 and 0.62 , respectively, and there was a higher sensitivity $(0.88$ and 0.79 ) at the expense of low specificity (0.38 and 0.41) for these thresholds (see Fig. 1a, b). For pre-recanalization SBP and MAP, 163 and $117 \mathrm{~mm} \mathrm{Hg}$ were identified as thresholds, respectively, with lower BP values predicting an mRS score of 0-2 (see Fig. 1c, d). Here,

Table 2 BP variables

\begin{tabular}{|c|c|c|c|c|}
\hline Phase & Mean SBP (SD) (mm Hg) & Mean DBP (SD) (mm Hg) & Mean MAP (SD) (mm Hg) & $n$ \\
\hline Baseline & $169.5(24.5)$ & $92.7(15.7)$ & $118.3(16.5)$ & 136 \\
\hline Pre-recanalization & $155.4(19.1)$ & $84.1(11.3)$ & $107.0(12.5)$ & 139 \\
\hline Post recanalization & $151.4(22.6)$ & $80.6(13.7)$ & $104.2(15.0)$ & 136 \\
\hline Post intervention & $147.2(23.3)$ & $76.7(13.7)$ & $100.2(15.5)$ & 137 \\
\hline Phase & $\triangle \mathrm{SBP}(\mathrm{SD})(\mathrm{mm} \mathrm{Hg})$ & $\triangle \mathrm{DBP}(\mathrm{SD})(\mathrm{mm} \mathrm{Hg})$ & $\triangle \mathrm{MAP}(\mathrm{SD})(\mathrm{mm} \mathrm{Hg})$ & $n$ \\
\hline Baseline to pre-recanalization & $-13.9(23.0)$ & $-8.5(15.4)$ & $-10.3(16.5)$ & 136 \\
\hline Baseline to post recanalization & $-18.0(26.7)$ & $-12.0(16.3)$ & $-14.0(18.3)$ & 136 \\
\hline Baseline to post intervention & $-22.3(29.4)$ & $-16.0(17.4)$ & $-18.1(19.6)$ & 136 \\
\hline
\end{tabular}

BP data of 3 patients during baseline and 1 patient during post intervention were missing

$\mathrm{BP}$, blood pressure, DBP, diastolic blood pressure, MAP, mean arterial pressure, SBP, systolic blood pressure, SD, standard deviation 
Table 3 Association of BP with change of NIHSS score after $24 \mathrm{~h}$

\begin{tabular}{|c|c|c|}
\hline Phase & $95 \% \mathrm{Cl}$ & $P$ \\
\hline \multicolumn{3}{|l|}{ SBP during different phases } \\
\hline Baseline & 0.120 .04 to 0.19 & 0.002 \\
\hline Pre-recanalization & 0.180 .08 to 0.29 & $<0.001$ \\
\hline Post-recanalization & 0.120 .03 to 0.20 & 0.006 \\
\hline Postintervention & $0.07-0.01$ to 0.16 & 0.103 \\
\hline \multicolumn{3}{|l|}{$\triangle S B P$ between different phases } \\
\hline From baseline to pre-recanalization & $0.01-0.08$ to 0.10 & 0.759 \\
\hline $\begin{array}{l}\text { From baseline to post-recanali- } \\
\text { zation }\end{array}$ & $0.03-0.10$ to 0.16 & 0.663 \\
\hline From baseline to postintervention & $0.05-0.02$ to 0.12 & 0.134 \\
\hline \multicolumn{3}{|l|}{ DBP during different phases } \\
\hline Baseline & $-0.08-0.21$ to 0.04 & 0.185 \\
\hline Pre-recanalization & $-0.19-0.36$ to -0.02 & 0.029 \\
\hline Post-recanalization & $-0.12-0.26$ to 0.02 & 0.082 \\
\hline Postintervention & $-0.13-0.28$ to 0.01 & 0.076 \\
\hline \multicolumn{3}{|l|}{$\triangle \mathrm{DBP}$ between different phases } \\
\hline From baseline to pre-recanalization & $0.02-0.12$ to 0.15 & 0.812 \\
\hline $\begin{array}{l}\text { From baseline to post-recanali- } \\
\text { zation }\end{array}$ & $-0.01-0.10$ to 0.08 & 0.845 \\
\hline From baseline to postintervention & $0.02-0.11$ to 0.14 & 0.781 \\
\hline \multicolumn{3}{|l|}{ MAP during different phases } \\
\hline Baseline & 0.090 .00 to 0.18 & 0.063 \\
\hline Pre-recanalization & $0.08-0.04$ to 0.21 & 0.176 \\
\hline Post-recanalization & $0.05-0.05$ to 0.15 & 0.344 \\
\hline Postintervention & $-0.01-0.11$ to 0.09 & 0.807 \\
\hline \multicolumn{3}{|l|}{$\triangle M A P$ between different phases } \\
\hline From baseline to pre-recanalization & $0.03-0.06$ to 0.13 & 0.492 \\
\hline $\begin{array}{l}\text { From baseline to post-recanali- } \\
\text { zation }\end{array}$ & $0.04-0.05$ to 0.12 & 0.423 \\
\hline From baseline to postintervention & 0.090 .01 to 0.17 & 0.036 \\
\hline
\end{tabular}

Depicted values are the results of multivariable linear regression analyses, which were performed separately for each phase. Several covariables were used for adjustment and are omitted here for clarity. The full regression models are shown in Supplementary Tables 5 and 6 . $\beta$ depicts the change of NIHSS score after $24 \mathrm{~h}$ per $\mathrm{mm} \mathrm{Hg}$ increase

$\mathrm{BP}$, blood pressure, DBP, diastolic blood pressure, MAP, mean arterial pressure, NIHSS, National Institutes of Health Stroke Scale, SBP, systolic blood pressure

sensitivity (0.68 and 0.78$)$ was also better at the cost of low specificity ( 0.43 and 0.34 ), with areas under the curves of 0.563 and 0.562 .

\section{Discussion}

The main findings of the study are as follows: (1) Higher SBP during the baseline and pre-recanalization phases was associated with reduced early neurologic recovery and an unfavorable long-term 3-month mRS score. Higher MAP during the baseline and pre-recanalization phases was also associated with an unfavorable 3-month $\mathrm{mRS}$ score. Thresholds discriminating favorable and
Table 4 Association of BP with mRS score 0-2 after 3 mo

\begin{tabular}{|c|c|c|c|}
\hline Phase & OR & $\begin{array}{l}95 \% \\
\text { confidence } \\
\text { interval }\end{array}$ & $P$ \\
\hline \multicolumn{4}{|l|}{ SBP during different phases } \\
\hline Baseline & 0.76 & $0.61-0.95$ & 0.015 \\
\hline Pre-recanalization & 0.65 & $0.47-0.91$ & 0.011 \\
\hline Post-recanalization & 0.84 & $0.66-1.06$ & 0.136 \\
\hline Postintervention & 0.85 & $0.66-1.08$ & 0.184 \\
\hline \multicolumn{4}{|l|}{$\triangle S B P$ between different phases } \\
\hline From baseline to pre-recanalization & 0.95 & $0.75-1.20$ & 0.642 \\
\hline From baseline to post-recanalization & 0.93 & $0.75-1.16$ & 0.529 \\
\hline From baseline to postintervention & 0.88 & $0.71-1.08$ & 0.222 \\
\hline \multicolumn{4}{|l|}{ DBP during different phases } \\
\hline Baseline & 1.12 & $0.78-1.61$ & 0.539 \\
\hline Pre-recanalization & 1.19 & $0.70-2.00$ & 0.523 \\
\hline Post-recanalization & 1.24 & $0.84-1.82$ & 0.274 \\
\hline Postintervention & 1.54 & $0.99-2.40$ & 0.055 \\
\hline \multicolumn{4}{|l|}{$\triangle \mathrm{DBP}$ between different phases } \\
\hline From baseline to pre-recanalization & 1.12 & $0.78-1.61$ & 0.548 \\
\hline From baseline to post-recanalization & 0.99 & $0.69-1.42$ & 0.939 \\
\hline From baseline to postintervention & 0.88 & $0.62-1.25$ & 0.464 \\
\hline \multicolumn{4}{|l|}{ MAP during different phases } \\
\hline Baseline & 0.76 & $0.58-1.00$ & 0.050 \\
\hline Pre-recanalization & 0.66 & $0.45-0.96$ & 0.030 \\
\hline Post-recanalization & 0.95 & $0.71-1.27$ & 0.715 \\
\hline Postintervention & 1.10 & $0.83-1.46$ & 0.499 \\
\hline \multicolumn{4}{|l|}{$\triangle M A P$ between different phases } \\
\hline From baseline to pre-recanalization & 1.02 & $0.78-1.33$ & 0.874 \\
\hline From baseline to post-recanalization & 0.90 & $0.70-1.14$ & 0.376 \\
\hline From baseline to postintervention & 0.75 & $0.58-0.98$ & 0.032 \\
\hline
\end{tabular}

Depicted values are the results of multivariable logistic regression analyses, which were performed separately for each phase. Several covariables were used for adjustment and are omitted here for clarity. The full regression models are shown in Supplementary Tables 8 and 9. OR depicts the change of odds for mRS score $0-2$ after $3 \mathrm{mo}$ per $10 \mathrm{~mm} \mathrm{Hg}$ increase

$\mathrm{BP}$, blood pressure, DBP, diastolic blood pressure, MAP, mean arterial pressure, mRS, modified Rankin Scale, OR, odds ratio, SBP, systolic blood pressure

unfavorable functional outcomes were identified as 187 and $125 \mathrm{~mm} \mathrm{Hg}$ for SBP and MAP on admission, respectively. For the intraprocedural pre-recanalization phase, thresholds of 163 and $117 \mathrm{~mm} \mathrm{Hg}$ for SBP and MAP, respectively, were found. (2) Reductions or changes in $\mathrm{BP}$ from baseline to the pre-recanalization phase of EST showed no association with functional outcome.

\section{Association of High Pre-recanalization BP with Worse Functional Outcome}

An association of higher mean intraprocedural pre-recanalization SBP and MAP with unfavorable functional 

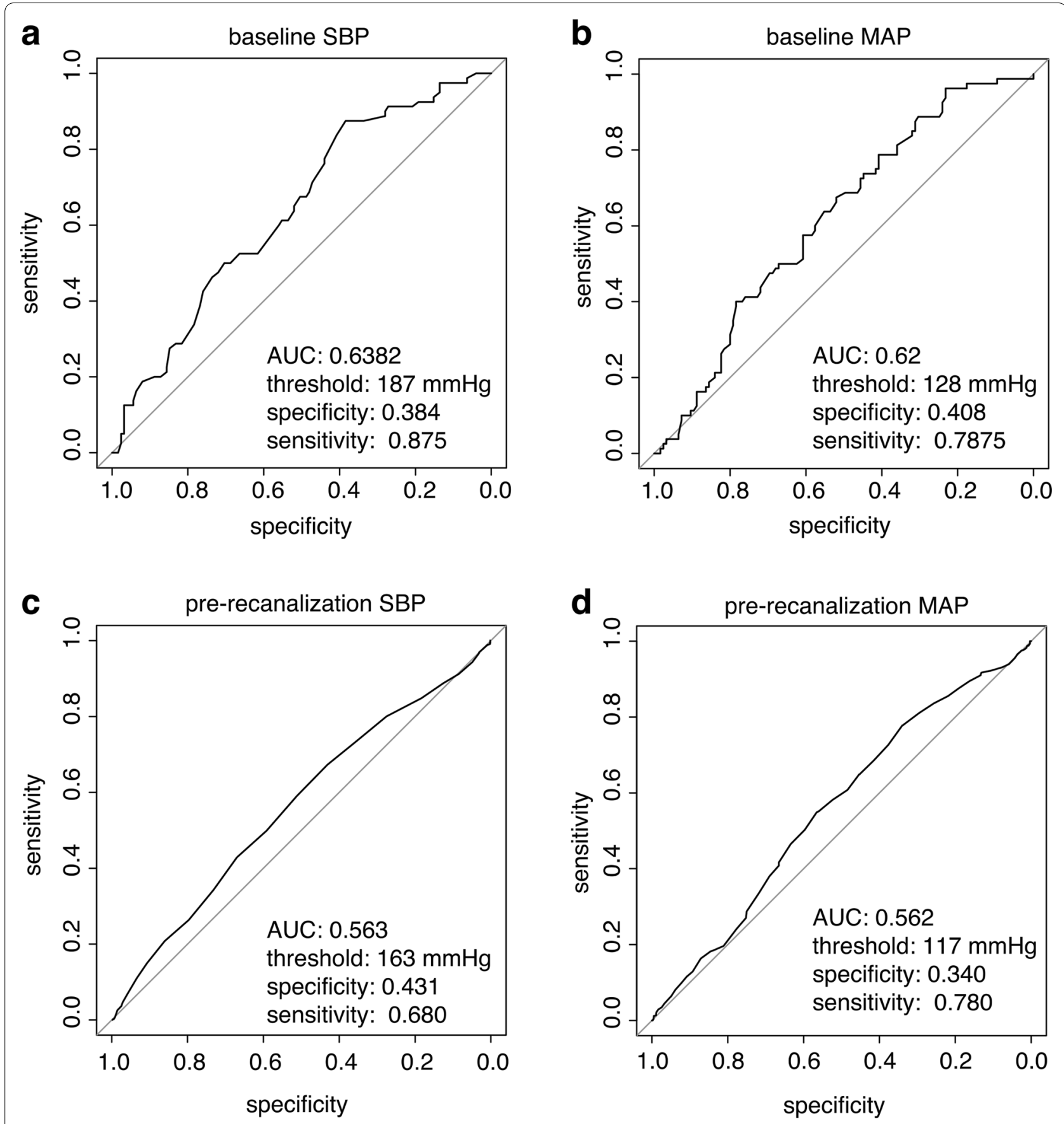

Fig. 1 Receiver operating characteristic curves of different blood pressure values at baseline $(\mathbf{a}, \mathbf{b})$ and during pre-recanalization $(\mathbf{c}, \mathbf{d})$ and their sensitivity and specificity to predict a good functional outcome (mRS 0-2) are shown. AUC area under the curve, MAP mean arterial pressure, $m R S$ modified Rankin Scale, SBP systolic blood pressure

outcome was found in our study. These findings are not straightforward to interpret, as they are in line with some previous reports but in contrast with another study.

An all stroke U-shaped relationship between BP and outcome has been described for the admission BP, which includes an association of very high BP with a worse outcome $[5,6]$. Our statistical approach did not aim at finding a U-shaped relationship, but we also found a worse functional outcome with higher SBP and MAP; this is probably a sign of stroke severity with more unfavorable 
prognostic factors, such as worse collaterals [18, 19], in which the elevated systemic BP might constitute a compensatory mechanism in maintaining cerebral perfusion.

On the other hand, Pikija et al. [20] showed that higher intraprocedural SBP and MAP (e.g., more than 120\% compared with the preprocedure values) led to higher odds of favorable functional outcome. However, their cohort consisted of patients who were treated in general anesthesia, and patients who were unsuccessfully recanalized (TICI grade $0-2 b$, rate $26.2 \%$ ) were included in their analysis. A further major difference was that their patients had a lower average SBP on admission (150 vs. $170 \mathrm{~mm} \mathrm{Hg}$ ) and during the procedure (128 vs. $155 \mathrm{~mm}$ $\mathrm{Hg}$ ) compared with our cohort, as well as a lower MAP (107 vs. $118 \mathrm{~mm} \mathrm{Hg}$ on admission and 91 vs. $107 \mathrm{~mm} \mathrm{Hg}$ intraprocedurally) [20].

Taken together, BP may affect functional outcome depending on the $\mathrm{BP}$ range that the patients were exposed to. If SBP and MAP of patients are situated in a high range, such as in our cohort, higher values might be detrimental for functional outcome, as opposed to patients with $\mathrm{BP}$ in lower ranges.

\section{Intraprocedural Pre-recanalization BP Reductions and Functional Outcome}

Most retrospective studies suggested that intraprocedural BP reductions during EST might be associated with worse functional outcome [20-26]. This was mostly interpreted as the importance of maintaining the physiologically elevated BP in the face of large-vessel occlusion to preserve collateral perfusion.

In contrast with these studies, BP reductions were not associated with early or long-term neurological outcome in our study.

Our study cohort differed from those of the other studies in several aspects. Many of the studies mentioned have performed EST nonuniformly under general anesthesia (GA) and mechanical ventilation, which may have led to poorer functional outcome via associations with poor prognostic factors in addition to hypotension (e.g., GA rates of $35 \%$ [22] to $100 \%$ [21, 24]). However, even patients in whom EST was exclusively performed under procedural sedation, a decrease of $10 \%$ or more in MAP from baseline was still associated with poor functional outcome in one study [23]. The latter study had no formal BP protocol, patients were slightly more severely afflicted than in our study (NIHSS score 17 vs. 14, respectively), and average admission BP was situated in a slightly lower range (SBP 158 vs. $170 \mathrm{~mm} \mathrm{Hg}$ and MAP 107 vs. $118 \mathrm{~mm}$ $\mathrm{Hg})$ [23].

Lower admission SBP or MAP (e.g., admission SBP of 140 [24] and 147 [25] vs. $170 \mathrm{~mm} \mathrm{Hg}$ in our cohort, MAP of 107 [21] vs. $118 \mathrm{~mm} \mathrm{Hg}$ in our cohort) was also observed in the other retrospective studies, showing a detrimental association between BP reductions and functional outcome. Furthermore, average SBP or MAP during the EST in other studies was also lower than in our study cohort (e.g., procedural SBP of 144 [22] and 119 [24] vs. $155 \mathrm{~mm} \mathrm{Hg}$ in our cohort and MAP of 75-77 [21] vs. $107 \mathrm{~mm} \mathrm{Hg}$ in our cohort).

We speculate that in our study, hypotensive BP was counteracted more stringently than in the other studies, and maintaining BP was easier because we mainly treated patients with procedural sedation.

The relationship of BP and BP changes with functional outcome may depend on the exposed BP range. Our findings of associations of worse outcomes with higher intraprocedural SBP, but not with BP reductions, in contrast with the findings of other studies [20-26], possibly support a hypothesis that the type of relationship between BP and functional outcome may depend on which BP range patients are exposed to. Because the $\mathrm{BP}$ values of our patients were set in a rather high range, they might represent a subgroup of patients situated in the right ascending part of a possible U-shaped BP-functional outcome relationship, which was described for the admission BP [5, 6]. In a recent meta-analysis of BP data from three randomized clinical trials assessing the anesthetic strategy during EST [SAGA trial team: SIESTA, Anesthesia During Stroke (ANSTROKE), and General or Local Anesthesia in Intra Arterial Therapy (GOLIATH)] [27], prolonged intraprocedural high or low BP was associated with poor functional outcome, further supporting a U-shaped nonlinear relationship of BP and outcome during the intraprocedural phase of EST.

Our findings are especially relevant in the setting of procedural sedation or monitored anesthetic care, in which hypotension is less of a problem than with GA regimens. In our study, we found thresholds of SBP and MAP of 163 and $117 \mathrm{~mm} \mathrm{Hg}$, respectively, during the pre-recanalization phase to predict functional outcome, with higher values predicting poor functional outcome. It might be prudent that neurointensivists and neuroanesthesiologists not only pay attention for hypotensive drops but also avoid extremely high BP in patients during EST. However, actual thresholds for harm have to be evaluated in randomized controlled trials, and further studies are needed to dissect the nonlinear relationship between BP and outcome.

On a further note, pursuing absolute BP targets without considering the differences between patients with strokes may be a suboptimal approach, and research on the best individual BP is necessary. It would be interesting for future investigations to study whether each patient has their own $\mathrm{BP}$-outcome relationship during EST with an individual optimal BP corridor because 
of interindividual differences in occlusion locations, penumbra sizes, collateral status, and other patient-specific characteristics. A randomized trial to investigate an individual approach on optimal intraprocedural BP management has begun recruiting patients (ClinicalTrials.gov identifier NCT04578288) [28].

Limitations of this study comprise the small sample size, the monocentric and retrospective nature of the investigation, and the amount of statistical analysis performed, which limit the rigor of the evidence and can only allow for generating hypotheses that have to be confirmed in randomized controlled studies. Furthermore, $\mathrm{BP}$ values were obtained from noninvasive BP measurements and might deviate from invasive arterial measurements. In the analysis of the postinterventional phase, only the first three measured BP values were analyzed. Lastly, observed effect sizes were very small and are, hence, questionable regarding their clinical relevance.

Strengths of this study are the extensive and detailed BP data for different time points in the periinterventional setting of patients with acute ischemic stroke receiving EST. Furthermore, the association of BP and functional outcome was investigated in a homogenous group of patients, with the primary intention to perform EST with a procedural sedation regimen, thus minimizing the influence of GA as a confounder.

\section{Conclusions}

This exploratory study finds detrimental associations between high SBP and MAP during the pre-recanalization phase and functional outcomes after endovascular therapy. The threshold for harm needs further examination in future randomized controlled trials.

\section{Supplementary Information}

The online version contains supplementary material available at https://doi. org/10.1007/s12028-021-01229-w.

\section{Author details \\ ${ }^{1}$ Department of Neurology, Heidelberg University Hospital, Heidelberg, Ger- many. ${ }^{2}$ Institute of Medical Biometry and Informatics, Heidelberg University, Heidelberg, Germany. ${ }^{3}$ Department of Neuroradiology, Heidelberg University Hospital, Heidelberg, Germany. ${ }^{4}$ Department of Neurology, Kassel General Hospital, Kassel, Germany.}

\section{Author contributions}

MC, DK, SilS, JB designed the study. MC, SilS, SimS, JP, LJ collected the data. MC and DK performed statistical analyses. MC, DK, JB, MK, MAM, PAR, MB and SilS interpreted the data. MC, DK and SilS provided input for the final version of the manuscript. All authors reviewed the manuscript and provided editorial feedback.

\section{Souce of support}

Open Access funding enabled and organized by Projekt DEAL. The authors received no funding.

\section{Conflicts of interest}

$J P$ has received travel and meeting expenses from Stryker and MicroVention. $M B$ has received grants and personal fees from Novartis, Guerbet, and Codman; personal fees from Vascular Dynamics, Roche, Teva, Springer, Grifols, and Bayer Vital; and grants from the European Union, Siemens, Hopp Foundation, and the German Research Council (Deutsche Forschungsgemeinschaft). MAM has received grants and personal fees from Medtronic and MicroVention, personal fees from Route92, grants and personal fees from Stryker, and grants from Balt, outside the submitted work. JB reports receiving speaker honoraria and travel support from Zoll, Medtronic, and Boehringer Ingelheim and an award from the Patient-Centered Outcomes Research Institute. The remaining authors have no conflicts to disclose.

\section{Ethical approval/informed consent}

The authors adhered to all ethical guidelines, and the study was approved by the local institutional review board (Ethikkommission Medizinische Fakultät Heidelberg, ID S-325/2015).

\section{Open Access}

This article is licensed under a Creative Commons Attribution 4.0 International License, which permits use, sharing, adaptation, distribution and reproduction in any medium or format, as long as you give appropriate credit to the original author(s) and the source, provide a link to the Creative Commons licence, and indicate if changes were made. The images or other third party material in this article are included in the article's Creative Commons licence, unless indicated otherwise in a credit line to the material. If material is not included in the article's Creative Commons licence and your intended use is not permitted by statutory regulation or exceeds the permitted use, you will need to obtain permission directly from the copyright holder. To view a copy of this licence, visit http://creativecommons.org/licenses/by/4.0/.

\section{Publisher's Note}

Springer Nature remains neutral with regard to jurisdictional claims in published maps and institutional affiliations.

Received: 3 December 2020 Accepted: 6 March 2021

Published online: 17 June 2021

\section{References}

1. Talke PO, Sharma D, Heyer EJ, Bergese SD, Blackham KA, Stevens RD. Society for Neuroscience in Anesthesiology and Critical Care expert consensus statement: anesthetic management of endovascular treatment for acute ischemic stroke*: endorsed by the Society of Neurolnterventional Surgery and the Neurocritical Care Society. J Neurosurg Anesthesiol. 2014;26:95-108.

2. Fiehler J, Cognard C, Gallitelli M, et al. European recommendations on organisation of interventional care in acute stroke (EROICAS). Int J Stroke. 2016:11:701-16.

3. Powers WJ, Rabinstein AA, Ackerson T, et al. 2018 guidelines for the early management of patients with acute ischemic stroke: a guideline for healthcare professionals from the American Heart Association/American Stroke Association. Stroke. 2018;49:e46-110.

4. Turc G, Bhogal P, Fischer U, et al. European Stroke Organisation (ESO)European Society for Minimally Invasive Neurological Therapy (ESMINT) guidelines on mechanical thrombectomy in acute ischemic stroke. J Neurointerv Surg. 2019. https://doi.org/10.1136/neurintsurg-2018-014569.

5. Leonardi-Bee J, Bath PMW, Phillips SJ, Sandercock PAG. Blood pressure and clinical outcomes in the international stroke trial. Stroke. 2002:33:1315-20.

6. Mulder MJHL, Ergezen S, Lingsma HF, et al. Baseline blood pressure effect on the benefit and safety of intra-arterial treatment in MR CLEAN (Multicenter Randomized Clinical Trial of Endovascular Treatment of Acute Ischemic Stroke in the Netherlands). Stroke. 2017;48:1869-76.

7. Wallace JD, Levy LL. Blood pressure after stroke. JAMA. 1981;246:2177-80.

8. Britton M, Carlsson A, de Faire U. Blood pressure course in patients with acute stroke and matched controls. Stroke. 1986:17:861-4. 
9. Powers WJ, Rabinstein AA, Ackerson T, et al. Guidelines for the early management of patients with acute ischemic stroke: 2019 update to the 2018 guidelines for the early management of acute ischemic stroke: a guideline for healthcare professionals from the American Heart Association/American Stroke Association. Stroke. 2019;50:e344-418.

10. Schönenberger S, Weber D, Ungerer MN, et al. The KEEP SIMPLEST study: improving in-house delays and periinterventional management in stroke thrombectomy-a matched pair analysis. Neurocrit Care. 2019;31(1):46-55.

11. Schönenberger $S$, Uhlmann $L$, Hacke $W$, et al. Effect of conscious sedation vs general anesthesia on early neurological improvement among patients with ischemic stroke undergoing endovascular thrombectomy: a randomized clinical trial. JAMA. 2016;316:1986-96.

12. Davis MJ, Menon BK, Baghirzada LB, et al. Anesthetic management and outcome in patients during endovascular therapy for acute stroke. Anesthesiology. 2012;116:396-405.

13. Whalin MK, Lopian S, Wyatt K, et al. Dexmedetomidine: a safe alternative to general anesthesia for endovascular stroke treatment. J Neurointerv Surg. 2014;6:270-5.

14. John S, Hazaa W, Uchino K, et al. Lower intraprocedural systolic blood pressure predicts good outcome in patients undergoing endovascular therapy for acute ischemic stroke. Interv Neurol. 2016;4:151-7.

15. Martins Al, Sargento-Freitas J, Silva F, et al. Recanalization modulates association between blood pressure and functional outcome in acute ischemic stroke. Stroke. 2016;47:1571-6.

16. van Buuren S, Groothuis-Oudshoorn K. MICE: multivariate imputation by chained equations in R. J Stat Softw. 2011;45:56. https://doi.org/10.18637/ jss.v045.i03.

17. R Foundation for Statistical Computing. R: a language and environment for statistical computing, version 4.0.2 [computer program]. Vienna, Austria. R Foundation for Statistical Computing. 2020. http://www.R-proje ct.org.

18. Liebeskind DS, Jahan R, Nogueira RG, Zaidat OO, Saver JL, SWIFT Investigators. Impact of collaterals on successful revascularization in Solitaire FR with the intention for thrombectomy. Stroke. 2014;45:2036-40.
19. Lima FO, Furie KL, Silva GS, et al. The pattern of leptomeningeal collaterals on CT angiography is a strong predictor of long-term functional outcome in stroke patients with large vessel intracranial occlusion. Stroke. 2010;41:2316-22.

20. Pikija S, Trkulja V, Ramesmayer $C$, et al. Higher blood pressure during endovascular thrombectomy in anterior circulation stroke is associated with better outcomes. J Stroke. 2018;20:373-84.

21. Löwhagen Hendén P, Rentzos A, Karlsson JE, et al. Hypotension during endovascular treatment of ischemic stroke is a risk factor for poor neurological outcome. Stroke. 2015;46:2678-80.

22. Petersen NH, Ortega-Gutierrez S, Wang A, et al. Decreases in blood pressure during thrombectomy are associated with larger infarct volumes and worse functional outcome. Stroke. 2019;50:1797-804.

23. Whalin MK, Halenda KM, Haussen DC, et al. Even small decreases in blood pressure during conscious sedation affect clinical outcome after stroke thrombectomy: an analysis of hemodynamic thresholds. AJNR Am J Neuroradiol. 2017;38:294-8.

24. Treurniet KM, Berkhemer OA, Immink RV, et al. A decrease in blood pressure is associated with unfavorable outcome in patients undergoing thrombectomy under general anesthesia. J Neurointerv Surg. 2018;10(2):107-11.

25. Valent A, Sajadhoussen A, Maier B, et al. A 10\% blood pressure drop from baseline during mechanical thrombectomy for stroke is strongly associated with worse neurological outcomes. J Neurointerv Surg. 2020;12:363-9.

26. Maïer B, Fahed R, Khoury N, et al. Association of blood pressure during thrombectomy for acute ischemic stroke with functional outcome: a systematic review. Stroke. 2019;50:2805-12.

27. Rasmussen $\mathrm{M}$, Schönenberger $\mathrm{S}$, Hendèn $\mathrm{PL}$, et al. Blood pressure thresholds and neurologic outcomes after endovascular therapy for acute ischemic stroke. JAMA Neurol. 2020;77(5):622-31.

28. Individualized blood pressure management during endovascular stroke treatment (INDIVIDUATE). ClinicalTrials.gov. National Library of Medicine, Bethesda (MD). 2021. http://clinicaltrials.gov/ct2/show/NCT04578288. Accessed 10 Feb 2021. 